\title{
La teoría de la complejidad en Biblioteconomía y Documentación: análisis y presencia en la investigación
}

\author{
Complexity Theory in Library and Information Science: a state of the art
}

\section{Sara Martínez Cardama, Mercedes Caridad Sebastián}

Universidad Carlos III de Madrid, Departamento de Biblioteconomía y Documentación, Instituto Agustín Millares, C/ Madrid 126, Getafe (Madrid, España), smarti1@bib.uc3m.es, mercedes@bib.uc3m.es

\begin{abstract}
Resumen
A través del vaciado de las bases de datos The $L i$ brary Science Database Library \& Library Information Science Abstracts database (LISA), se proporciona el análisis e implicaciones del uso de la Complejidad y la Teoría del Caos en el área de Biblioteconomía y Documentación. Tras una introducción a estos fundamentos epistemológicos, se analiza la producción científica en términos formales de autoría, filiaciones institucionales y principales revistas, y se categorizan temáticamente el total de 303 artículos que respondían a los criterios de investigación aplicados. Los resultados arrojan un uso limitado de estas teorías en el ámbito de la Biblioteconomía y Documentación, generalmente enmarcadas en las perspectivas de la gestión y comportamiento organizacional. Se incluyen vías de investigación posteriores.
\end{abstract}

Palabras clave: Teoría de la complejidad. Teoría del caos. Fundamentación teórica. Biblioteconomía y Documentación.

\section{Introducción}

El objetivo de la presente investigación es identificar y definir los acercamientos a las teorías de la Complejidad y el Caos desde el ámbito de la Biblioteconomía y Documentación (Library and Information Science).

La presencia de teorías en las investigaciones, ayudan tanto a su respaldo y validación como a organizar y comunicar datos y simplificar la complejidad de los acontecimientos del mundo social (Van Maanen, 1988). Su presencia es considerada por la Filosofía de la Ciencia como un estadio de madurez en cualquier investigación (Brookes, 1980).Esta idea entronca a la perfección con la defensa del pensamiento complejo en la investigación teórica, entendido como un marco conceptual en el que situar proyectos más que como una metodología o un conjunto de herramientas (Middleton-Kelly, 2003). Esta manera de ver el mundo que constituyen las Ciencias de la Complejidad y las implicaciones

\begin{abstract}
This state of the art on is based on the Library Science Database Library and the Library Information Science Abstracts database (LISA), and provides an analysis and the implications of the use of Complexity and Chaos Theory in the field of Library and Information Science. After an introduction to its epistemological foundations, we analyze the scientific production in formal terms of authorship, institutional affiliations and main journals, as well as a thematic categorization of a total of 303 articles that met the applied research criteria. The results show a limited use of these theories in the field of Library and Information Science, which is generally framed by the perspectives of Management and Organizational Behaviour. Suggestions for further research are also included.
\end{abstract}

Keywords: Complexity Theory. Chaos Theory. Theoretical research. Library and Información Science.

de la Teoría del Caos no es unificada: se ha empleado de manera transversal desde ramas diversas del conocimiento, especialmente desde la Gestión y la perspectiva organizacional, la Informática, la Computación, la Física o la Educación. Si bien se darán los elementos clave con el fin de poder enmarcar estas teorías, el presente artículo se centra en su confluencia en el ámbito de la Biblioteconomía y Documentación.

Durante años se destacó la ausencia de un corpus teórico sólido en el ámbito de la Biblioteconomía y Documentación. Esta falta de "teorías" fue puesta de manifiesto por Hjørland (1998). Sin embargo, Pettigrew y Mackechnie concluyeron en 2001 que existía una presencia del uso de teorías en el $34,1 \%$ de los artículos del área LIS, con marcada tendencia ascendente; aunque reconocen un escaso debate y citación de las aportaciones teóricas en los 1160 artículos analizados entre 1993 y 1998.

Si bien la de la Complejidad no constituye una teoría unificada que pueda compararse con otras 
utilizadas en nuestro campo, permite un enfoque de estudio diferente a problemas de nuestro ámbito disciplinar. Así, el reto digital, la transformación híbrida de los centros de información, los cambios en su gestión pueden ser entendidos desde este marco que concibe la complejidad como una propiedad inherente a los sistemas.

La interdisciplinariedad está presente como base para muchas disciplinas; así, por ejemplo, en las teorías económicas que van de la mano de conceptos filosóficos (Teoría de la Racionalidad, la Teoría de Juegos, de la Incertidumbre...). En cuanto al ámbito de la Biblioteconomía y Documentación, su estructura es en sentido amplio interdisciplinar (Saracevic, 1995). Nolin y Aström (2010) llegan a habkar de "una naturaleza fragmentada", algo que estos autores consideran como un elemento de valor añadido, frente a características anteriormente negativas como el difícil establecimiento de límites y la ausencia de un núcleo disciplinar claro. Cronin (2012) vuelve a incidir en esta idea al mencionar el carácter permeable del ámbito LIS hacia otras disciplinas, constituyendo un núcleo de competencias y saberes cada vez menos homogéneo.

Este trabajo tiene su origen en el marco teórico de la tesis doctoral de Martínez Cardama (2014) en el que se definía la complejidad como elemento vehicular para estudiar el concepto de biblioteca digital universitaria. Se considera ahora oportuno el estudio de su presencia en la investigación del ámbito LIS para valorar finalmente su potencial importancia y utilización en un área como las Ciencias de la Documentación, que está siendo constantemente reformulada en cuanto a sus límites y campo temático (García Marco, 2013; Bereijo Martínez, 2012).

\subsection{Las Ciencias de la Complejidad y la Teoría del Caos: una introducción}

Siguiendo el criterio kuhniano de "Ciencia Normal", entendiendo esta como el período en el que la ciencia y las ideas se enmarcan en un estado general teórico definido, las Ciencias de la Complejidad suponen una nueva visión para afrontar los problemas científicos. La Complejidad actúa como elemento transversal en la Ciencia actual. Supone un mecanismo innovador de estudio, en particular del estudio de los procesos de interacción en sistemas socio-técnicos como las organizaciones, por ejemplo. Constituye una propiedad emergente de estos sistemas, compuestos a su vez por elementos caracterizados por su autoorganización y la no linealidad (Gilpin y Murphy, 2008; Lyytinen y Newman, 2008).

A tenor de González (2013), existen formas estructurales de complejidad y aspectos dinámi- cos de la misma, que, en principio, rebasan las propuestas de una serie dominante de métodos en una ciencia específica. Las emergentes Ciencias de la Complejidad ofrecen una alternativa al estudio de las redes de interacciones que suponen a nivel global los diferentes sistemas económicos, sociales, tecnológicos y ecológicos (Heylighen, Cilliers y Gershenson, 2006).

Estas crisis interpretativas científicas se hacen profundas en las Ciencias Sociales: la aparición de las nuevas tecnologías y sus consecuencias socio-culturales rompen conceptos tradicionales de certidumbre. Por lo tanto, la complejidad es un elemento inherente y necesario para el estudio de cualquier aspecto en la sociedad actual.

La detección y gestión de la complejidad supone un reto para su integración en los estándares científicos, siendo esto más complejo en Ciencias Sociales, y no así en las Ciencias de la Vida, donde tiene amplia tradición apoyada en un lenguaje formal, al igual que en Computación (Rodríguez Zoya y Aguirre, 2011). Así, Heylighen, Cilliers y Gershenson (2006) reconocen un uso muy especializado de la Complejidad aplicada a ámbitos reducidos como los algoritmos de clustering, las simulaciones en computadoras o las ecuaciones no lineales. Por el contrario, a nivel terminológico su uso es frecuente para delimitar marcos teóricos o incluir metáforas determinadas para calificar y describir ciertos sucesos. Así, palabras como "emergencia", "no linealidad", "autoorganización" o "caos y ausencia de equilibrio" se suceden en las investigaciones sin constituir un corpus teórico uniforme.

Históricamente, el interés por la Complejidad como elemento de análisis en la Filosofía de la Ciencia ha sido una constante a lo largo del siglo XX, desde el Círculo de Viena a Popper (Rodríguez Zoya; Leónidas Aguirre, 2011). Aunque históricamente, según Delic y Dum (2006), su interés comienza a incrementarse en la II Guerra Mundial, debido a la búsqueda de optimización de los sistemas y simulaciones en el campo de la tecnología atómica.

Estos autores fijan la primera aproximación conceptual en un artículo científico firmado por Weaver en 1948 con el título Science and Complexity. En este trabajo, Weaver comienza a establecer tres problemas científicos: la simplicidad (problemas que pueden resolverse con pocas variables), complejidad desorganizada (resuelta mediante mecanismos estadísticos) y la complejidad organizada. Weaver califica de complejidad organizada a los fenómenos donde intervienen un amplio número de factores que forman un todo. Esa totalidad se resuelve mediante modelos sistémicos. 
Es por esta razón que el estudio de la complejidad se aborda desde la perspectiva de los sistemas, especialmente gracias a la aparición de la Teoría de Sistemas de Von Bertalanffy que promulgó la existencia de sistemas abiertos señalando la presencia de límites o fronteras. Posteriormente, para mostrar las relaciones entre los subsistemas, la Cibernética estudiará los procesos de comunicación. Maturana y Varela (1979) acuñaron en este contexto los conceptos de autonomía y autopoiesis para diferenciar a los sistemas vivos de los artificiales, ligados al ámbito de la Ingeniería y las Ciencias de lo Artificial y desarrollados especialmente por Herbert A. Simon en su trabajo titulado "La arquitectura de la Complejidad", donde establece una distinción de estructura jerárquica de estos sistemas complejos.

Es en los años 80 cuando aparecen los conceptos de Sistemas Adaptativos Complejos y de manera más general el término "Ciencias de la Complejidad". Heylighen, Cilliers y Gershenson (2006) resumen las raíces de este último aporte de la Complejidad: la dinámica no lineal, la Computación y la simulación, el estudio de formas complejas a través de la Biología, y la aplicación de esos métodos al estudio de sistemas sociales desde los mercados bursátiles o Internet donde no existe un orden predefinido. Estos mismos autores reconocen que a nivel filosófico, los autores que abordan la Teoría de la Complejidad como parte de sus investigaciones no acuden a planteamientos filosóficos, salvo en el caso de la Cibernética o la Teoría de Sistemas.

En esta década, la Teoría de los Sistemas Complejos se abre paso en las Ciencias Sociales. Uno de sus artífices, Luhmann (1998), la dota de propiedades de "metateoría" que atraviesa ciencias muy dispersas entre sí, en definitiva, una manera de ver el mundo, desde la Economía hasta aspectos sociales.

Sin embargo, una de las dificultades de la traslación de la Teoría de la Complejidad a las Ciencias Sociales es, a tenor de Heylighen, Cilliers y Gershenson (2006), la influencia de la Teoría del Caos y de la Escuela de Santa Fe. Sus usos a nivel de terminología son similares a los de la Complejidad y su estudio se centra en la no-linealidad de los sistemas dinámicos (Gleick, 1987), es decir, aquellos en los que la relación entre sus variables es no lineal. En sentido estricto, el estudio de la Complejidad suele abarcar tres tipos de sistemas: las estructuras disipativas, los sistemas caóticos y lo que es entendido como sistemas complejos (Gildtrap, 2009). El presente estudio ha podido comprobar cómo las investigaciones suelen utilizar la Teoría del Caos y la Complejidad de manera muchas veces conjunta, como elementos críticos de cualquier sistema.

Para concluir este apartado es preciso señalar, a partir del estudio teórico, la progresiva separación en el uso de la Complejidad en la literatura. Así, Woermann (2016) establece, por un lado, la llamada Filosofía de la Complejidad (asociada a la obra crítica de Cilliers y Morin, por ejemplo) y otro uso más taxativo de la misma, la llamada Complejidad científica, asociada a las teorías de la Escuela de Santa Fe que busca los principios de los sistemas físicos y computaciones para su posterior modelización.

\subsection{Las Ciencias de la Documentación y la Complejidad}

Para Bereijo Martínez (2012), la red de interdependencias que constituyen los elementos de archivos, bibliotecas y centros de documentación es clave para abordar el problema de la complejidad, especialmente en el contexto digital, fuertemente dinámico.

Desde el estudio teórico del concepto de Información, Bawden y Robinson (2015) utilizan la complejidad como un elemento para su definición. Así, la Información se concibe dentro de una perspectiva compleja, enmarcándola en conceptos como el orden y la organización, pero también el caos y la entropía, siguiendo las teorías de Shannon y Weaver (1949). Contrasta esta visión con la de Wiener (1948) sobre la información como estructura, como medida de reducción del caos.

El mismo Bawden en 2007, ya había estudiado el concepto unificado de información como sistema auto-organizativo complejo en los dominios de la Física, la Biología y las Ciencias Humanas. La complejidad le ayuda al autor a ofrecer una definición de información que sea un puente entre varias disciplinas, y que, a su vez la Information Science pueda servir de nicho conceptual a otros campos de conocimiento.

Este carácter dual sobre la complejidad en el concepto de la Información (Estructura vs. ausencia de orden), es válido en la formulación epistémica en las Ciencias de la Documentación, que como disciplina goza de elementos constitutivos estructurales (asociados en la investigación en temas como la organización y sistemas complejos); y una fuerte vertiente dinámica, marcada de manera profunda en su historicidad. Esta complejidad que caracteriza a los fenómenos del ámbito LIS, pero también a los fenómenos económicos o comunicativos, no puede abarcarse desde la perspectiva de un todo (González, 2012). 
La incursión en la teoría de la Complejidad desde la perspectiva organizacional en bibliotecas tiene diferentes enfoques. Si bien la Teoría de Sistemas fue ampliamente estudiada y contemplada como influencia (Ranganathan ya utilizaba esta perspectiva apelando al carácter orgánico de las bibliotecas), la aparición de las redes de información supuso una renovación en la investigación en bibliotecas desde la perspectiva organizacional, consideradas como sistemas complejos (Gildtrap, 2009). También en el diseño de los programas formativos en LIS se debatió la inclusión de estos elementos (Grover et al., 1997), algo frecuente en programas de estudio del área de Gestión y Organización.

En cuanto a la vertiente más dinámica, la investigación destaca en sus inclinaciones hacia la web y el entorno digital. En este sentido, cabe señalar las aportaciones de la obra de Tredinnick (2009), Complexity theory and the web. Este autor define la Web (el discurso web) como un Sistema Adaptativo Complejo, que cumple las siguientes características: i) está compuesto por un largo número de elementos individuales; ii) estos elementos interactúan de manera dinámica; iii) las interacciones son de tipo no lineal y predominan las locales, entre elementos más próximos; iv) son sistemas abiertos (se adaptan al entorno); y v) operan lejos del equilibrio, se adaptan y evolucionan, a pesar de que en algún momento puedan ser estables.

Tredinnick define al entorno web como un Sistema Adaptativo Complejo que posee dos elementos definitorios en cuanto a su complejidad: la emergencia y la auto-organización. Ofrece un modelo conceptual y terminológico bajo esta perspectiva tanto para la Web tradicional (a la que Berners Lee asemejó con una simplificación por número de niveles), como para el estándar RDF y la Web 2.0. Este autor ve necesario continuar utilizando esta teoría para la modelización de la Web desde elementos como el Cloud Computing o la Web 3.0. Estas características sirven a nivel conceptual para definir el campo temático LIS, cuyos límites están constantemente ampliándose y viéndose afectados por su vertiente dinámica. Sin embargo, el uso de estos preceptos y conceptos no está presente en la investigación teórica a este nivel, como muestra este estudio.

\section{Metodología}

El presente estudio pretende detectar las influencias de la Teoría de la Complejidad a través de la investigación bibliográfica volcada en dos bases de datos del sector: The Library Science Database Library y Library Information
Science Abstracts database (LISA). Mediante esta investigación se trató de averiguar si existen sólidas conexiones entre la Biblioteconomía y Documentación y la presencia de la Complejidad como elemento teórico, bajo que perspectiva se aplica y a qué preguntas responde.

El estudio se realizó entre los meses de enero y abril de 2017, siendo consultadas ambas bases de datos de manera simultánea a través del proveedor Proquest. La ecuación de búsqueda planteada fue: (Librar* OR "Information Science") AND ("Complexity Theory" OR "Chaos Theory"). Se descartó excluir Theory pues la búsqueda por conceptos genéricos como Complejidad o Caos deformaba mucho los resultados. A pesar de señalar la inclusión del concepto del Caos en la Teoría de la Complejidad se decidió incluirlo debido a la gran influencia y uso que la "Teoría del Caos" tiene en solitario en la investigación. Tras la realización de la búsqueda se obtuvieron 365 ítems, de los que finalmente se valoraron 303 . El proceso de análisis, si bien aporta datos cuantitativos, fue de carácter cualitativo, y requirió la lectura detallada de cada artículo para ver el enfoque y adscripción temática de ambas teorías en cada una de las obras.

Una vez obtenido el conjunto de documentos se conformó una base de datos con los siguientes campos: título, autoría, filiación institucional, fecha y revista. Seguidamente, se procedió a su clusterización semántica a través de técnicas de análisis de contenido para detectar patrones en la muestra documental. Se excluyeron a priori las palabras clave automáticas o dadas por el autor. Se prefirió una redacción propia de las palabras clave partiendo de la definición de categorías temáticas principales. Primero, se trató de definir la disciplina o campo de conocimiento a la que se adscribían los artículos. Dado que muchas disciplinas pertenecen al ámbito de la Gestión empresarial se contó con ayuda de herramientas de clasificación como la JEL Classification de la American Economic Association para definirlas con mayor precisión. Finalmente, se definieron los subtemas o temas específicos que tratan los artículos.

Fruto tanto del análisis temático detallado como de la explotación de la base de datos creados, los resultados ofrecen información sobre los principales autores, su área de adscripción, así como las revistas en las que se publica, y el ámbito temático al que se adscriben los artículos. Con todo ello se podrá detectar la influencia real de estos fundamentos teóricos en el ámbito de Biblioteconomía y Documentación. 


\section{Resultados}

\subsection{Resultados de la actividad investigadora}

Al no haber limitado la búsqueda por fecha, puede apreciarse la evolución de la investigación (Tabla I). Se observa un incremento desde el año 2000 hasta nuestros días, representando la producción anterior a ese año solo un $11.2 \%$.

\begin{tabular}{rr}
\hline Año & Docs \\
\hline 1987 & 1 \\
\hline 1990 & 1 \\
\hline 1992 & 2 \\
\hline 1993 & 2 \\
\hline 1994 & 2 \\
\hline 1995 & 1 \\
\hline 1996 & 7 \\
\hline 1997 & 3 \\
\hline 1998 & 4 \\
\hline 1999 & 11 \\
\hline 2000 & 7 \\
\hline 2001 & 10 \\
\hline 2002 & 17 \\
\hline 2003 & 11 \\
\hline 2004 & 17 \\
\hline 2005 & 12 \\
\hline 2006 & 27 \\
\hline 2007 & 16 \\
\hline 2008 & 26 \\
\hline 2009 & 18 \\
\hline 2010 & 23 \\
\hline 2011 & 16 \\
\hline 2012 & 14 \\
\hline 2013 & 15 \\
\hline 2014 & 11 \\
\hline 2015 & 15 \\
\hline 2016 & 12 \\
\hline 2017 & 2 \\
\hline Total & 303 \\
\hline & \\
\hline 203
\end{tabular}

Tabla I. Distribución por fechas

\subsection{Resultados de la autoría de los trabajos}

Se contabilizaron 566 autores. De estos, 36 se calificaron como los más prolíficos: con más de una publicación por autor.

\begin{tabular}{lrl}
\hline Autores & $N$ & Filiación \\
\hline Lyytinen, Kalle & 5 & $\begin{array}{l}\text { Dept.of Information Systems, Weatherhead } \\
\text { School of Management }\end{array}$ \\
\hline Dumay, Jonhn C. & 4 & $\begin{array}{l}\text { Faculty of Economics and Business, The } \\
\text { University of Sydney }\end{array}$ \\
\hline Merali,Yasmin & 3 & $\begin{array}{l}\text { Warwick Business School, The University of } \\
\text { Warwick }\end{array}$ \\
\hline Vandergriff, Linda J. & 3 & The Aerospace Corporation \\
\hline $\begin{array}{l}\text { Al Saifi, Said } \\
\text { Abdullah }\end{array}$ & 2 & $\begin{array}{l}\text { International Business Administration, Nizwa } \\
\text { College of Applied Sciences }\end{array}$ \\
\hline Allee,Verna & 2 & Verna Allee Associates \\
\hline Backström,Tomas & 2 & $\begin{array}{l}\text { Dept. of Computer and Systems Sciences, } \\
\text { Stockholms Universitet }\end{array}$ \\
\hline
\end{tabular}

\begin{tabular}{|c|c|c|}
\hline Bawden, David & 2 & $\begin{array}{l}\text { Dept of Information Science, City University } \\
\text { London }\end{array}$ \\
\hline Benbya, Hind & 2 & UCLA Anderson School of Management \\
\hline Bennet, Alex & 2 & Mountain Quest Institute \\
\hline Bi, Zhuming & 2 & $\begin{array}{l}\text { Dept. of EngineeringIndiana University-Purdue } \\
\text { University Fort Wayne }\end{array}$ \\
\hline Browaeys, M. J. & 2 & $\begin{array}{l}\text { Center for Marketing \& Supply Chain } \\
\text { Management, Nyenrode Business Universiteit }\end{array}$ \\
\hline Fisser, $\mathrm{S}$. & 2 & Nyenrode Business Universiteit \\
\hline Governatori,Guido & 2 & $\begin{array}{l}\text { School of IT and Electrical Engineering. The } \\
\text { University of Queensland }\end{array}$ \\
\hline Hinz, O. & 2 & $\begin{array}{l}\text { Dept. of Strategy and Management, De } \\
\text { Montfort University }\end{array}$ \\
\hline Jones, Bonna & 2 & $\begin{array}{l}\text { School of Business Information Technology, } \\
\text { RMIT University }\end{array}$ \\
\hline Juntunen, Arja & 2 & University of Eastern Finland Library \\
\hline Kurtze, D. & 2 & Dept. of Physics, North Dakota State University \\
\hline Lankes, David & 2 & $\begin{array}{l}\text { Syracuse University's School of Information } \\
\text { Studies. }\end{array}$ \\
\hline Layzell Ward, P. & 2 & Editor, Library Management \\
\hline Line. M. B. & 2 & Editor,Alexandria \\
\hline McBride, $\mathrm{N}$. & 2 & $\begin{array}{l}\text { Faculty of Computing Sciences and } \\
\text { Engineering, De Montfort University }\end{array}$ \\
\hline McElroy, Mark W. & 2 & Macroinnovations Associates \\
\hline McKelvey, Bill & 2 & UCLA Anderson School of Management \\
\hline Mitleton-Kelly, Eve & 2 & London School of Economics \\
\hline Newman, Mike & 2 & $\begin{array}{l}\text { Norwegian School of Economics, and Business } \\
\text { Administration, Bergen }\end{array}$ \\
\hline Nicholson, Scott & 2 & AskScott \\
\hline Patel, Nandish V. & 2 & Brunel Business School \\
\hline Pienaar, $\mathrm{H}$. & 2 & $\begin{array}{l}\text { Academic Information Service, University of } \\
\text { Pretoria, Pretoria, South Africa. }\end{array}$ \\
\hline Rose, Gregory & 2 & College of Business, Washington State Univ. \\
\hline Saarti, Jarmo & 2 & University of Eastern Finland Library \\
\hline Sherif, Karma & 2 & $\begin{array}{l}\text { Dept of Business Administration, Texas } \\
\text { Southern University }\end{array}$ \\
\hline Smith, A. D. & 2 & $\begin{array}{l}\text { Dept of Management and Marketing, Robert } \\
\text { Morris University }\end{array}$ \\
\hline Snyder, H. & 2 & School of Library and Inf. Science, Indiana Univ. \\
\hline Tredinnick, Luke & 2 & $\begin{array}{l}\text { Dept of Applied Social Sciences, London } \\
\text { Metropolitan University }\end{array}$ \\
\hline Voigt, Dennis W & 2 & Accounting, Saint Michael's College \\
\hline
\end{tabular}

Tabla II. Autores más prolíficos y filiación institucional

Al analizar los datos puede verse que el $94 \%$ de las contribuciones se realizan por autores con solo una contribución. La Tabla II muestra los autores más prolíficos, indicando su vinculación institucional. A través de su filiación institucional, se detecta que los autores más prolíficos se vinculan a departamentos de Gestión, Empresa o Negocios en sus fórmulas más convencionales: Business Administration y Management. Esto se repite en el posterior análisis temático. Asimismo, también es de interés encontrar departamentos conjuntos sobre Empresa e IT o Ingeniería. Esto también se verá a la hora de analizar la temática de los artículos: en los que buena parte de los mismos cubren la convergencia entre el diseño de los sistemas de información y la empresa.

En cuanto a Biblioteconomía y Documentación solo figuran 6 autores dentro del listado de más 
prolíficos: dos de ellos, D Lankes y $\mathrm{H}$. Synder, a través de departamentos del área: la escuela de Information Studies de Syracuse y la Escuela de Information Science de Indiana. Las otras contribuciones se realizan a través de editoriales y revisiones de directores de revistas del área: $\mathrm{M}$. B. Line $(1995,2001)$ en Alexandria y W. Layzell (2000, 2002) en Library Management, quienes reflexionan sobre las tendencias más destacadas en Biblioteconomía y Documentación, entre las que incluyen la Teoría de la Complejidad en las organizaciones. Las otras dos autorías, J. Saarti y A. Juntunen (2011a, 2011b) y Pienaar (1999a, 1999b) se vinculan a bibliotecas universitarias, centros a los que más se ha aplicado la Teoría de la Complejidad en su gestión.

\begin{tabular}{lr}
\hline Filiación institucional & $N^{\circ}$ art. \\
\hline Empresa y Negocios (Business) & 77 \\
\hline Multidisciplinar & 52 \\
\hline Gestión (Management) & 37 \\
\hline Biblioteconomía y Documentación & 35 \\
\hline Ingeniería & 31 \\
\hline Economía, Finanzas y Contabilidad & 11 \\
\hline Comunicación & 10 \\
\hline Educación & 10 \\
\hline Informática, Computación & 8 \\
\hline Gestión del conocimiento & 7 \\
\hline Salud & 7 \\
\hline Gestión de la Información & 5 \\
\hline Arquitectura & 4 \\
\hline Psicología & 3 \\
\hline Derecho & 3 \\
\hline Ingeniería y Gestión & 1 \\
\hline Comercio & 1 \\
\hline Sistemas complejos & 1 \\
\hline
\end{tabular}

Tabla III. Filiación institucional total

Al analizar la filiación institucional que figura en la firma de los 303 artículos puede verse una relación muy similar en laTabla III. Los autores vinculados a departamentos de Gestión, Negocios, Empresa y Organización ocupan el 41,2\% del total de autores. En la categoría Multidisciplinar, se ha incluido tanto a autores que solo estaban vinculados con su universidad como aquellos a los que era difícil adscribirlos a un área determinada, por ejemplo: algunos de tipo mixto o genéricos como Ciencias Sociales, Tecnología y Cultura... En cuanto a departamentos exclusivos de LIS o instituciones bibliotecarias su presencia es del $11.5 \%$.

\subsection{Resultados de las revistas}

Se han recogido 89 títulos de revista. De estos, los títulos con al menos 2 publicaciones son las siguientes 35 (Tabla IV).

\begin{tabular}{|c|c|c|}
\hline Título & $N .^{\circ}$ & Tema \\
\hline The Learning Organization & 37 & $\begin{array}{l}\text { Recursos Humanos, } \\
\text { Aprendizaje y Estudios Org. }\end{array}$ \\
\hline $\begin{array}{l}\text { Journal of Knowledge } \\
\text { Management }\end{array}$ & 28 & $\begin{array}{l}\text { Gestión de la Información y el } \\
\text { Conocimiento }\end{array}$ \\
\hline $\begin{array}{l}\text { Journal of Information } \\
\text { Technology }\end{array}$ & 18 & $\begin{array}{l}\text { Biblioteconomía y Doc. (LIS) y } \\
\text { Sistemas de Información }\end{array}$ \\
\hline $\begin{array}{l}\text { Journal of Intellectual } \\
\text { Capital }\end{array}$ & 17 & $\begin{array}{l}\text { Gestión de la Información y el } \\
\text { Conocimiento }\end{array}$ \\
\hline VINE & 11 & $\begin{array}{l}\text { Gestión de la Información y el } \\
\text { Conocimiento }\end{array}$ \\
\hline $\begin{array}{l}\text { European Journal of } \\
\text { Information Systems }\end{array}$ & 9 & Computación \\
\hline $\begin{array}{l}\text { Information Systems } \\
\text { Frontiers }\end{array}$ & 9 & Educación \\
\hline $\begin{array}{l}\text { Information Technology \& } \\
\text { People }\end{array}$ & 9 & $\begin{array}{l}\text { Gestión de la Información y el } \\
\text { Conocimiento }\end{array}$ \\
\hline $\begin{array}{l}\text { Artificial Intelligence and } \\
\text { Law }\end{array}$ & 8 & Inteligencia artificial y Derecho \\
\hline Futurics & 8 & Prospectiva \\
\hline Library Management & 8 & $\begin{array}{l}\text { Biblioteconomía y } \\
\text { Documentación LIS) }\end{array}$ \\
\hline Corporate Communications & 6 & Comunicación corporativa \\
\hline $\begin{array}{l}\text { Journal of Communication } \\
\text { Management }\end{array}$ & 6 & Comunicación corporativa \\
\hline Journal of Documentation & 6 & $\begin{array}{l}\text { Biblioteconomía y } \\
\text { Documentación (LIS) }\end{array}$ \\
\hline $\begin{array}{l}\text { Journal of Enterprise } \\
\text { Information Management }\end{array}$ & 6 & $\begin{array}{l}\text { Biblioteconomía y } \\
\text { Documentación (LIS) }\end{array}$ \\
\hline $\begin{array}{l}\text { Journal of the American } \\
\text { Society for Inf. Science }\end{array}$ & 5 & $\begin{array}{l}\text { Biblioteconomía y } \\
\text { Documentación (LIS) }\end{array}$ \\
\hline Online Information Review & 5 & $\begin{array}{l}\text { Gestión de la Información y el } \\
\text { Conocimiento }\end{array}$ \\
\hline $\begin{array}{l}\text { The Artificial Intelligence } \\
\text { Review }\end{array}$ & 5 & Inteligencia Artificial \\
\hline Aslib Proceedings & 4 & $\begin{array}{l}\text { Gestión de la Información y el } \\
\text { Conocimiento }\end{array}$ \\
\hline $\begin{array}{l}\text { Journal of Global } \\
\text { Information Management }\end{array}$ & 4 & $\begin{array}{l}\text { Sistemas de Información y } \\
\text { Gestión en la Empresa }\end{array}$ \\
\hline $\begin{array}{l}\text { Journal of the American } \\
\text { Society for Information } \\
\text { Science and Technology }\end{array}$ & 4 & $\begin{array}{l}\text { Biblioteconomía y } \\
\text { Documentación (LIS) }\end{array}$ \\
\hline $\begin{array}{l}\text { Information Processing \& } \\
\text { Management }\end{array}$ & 3 & $\begin{array}{l}\text { Biblioteconomía y } \\
\text { Documentación (LIS) }\end{array}$ \\
\hline $\begin{array}{l}\text { Journal of Database } \\
\text { Management }\end{array}$ & 3 & Computación \\
\hline $\begin{array}{l}\text { Journal of Information } \\
\text { Science }\end{array}$ & 3 & $\begin{array}{l}\text { Biblioteconomía y } \\
\text { Documentación LIS) }\end{array}$ \\
\hline $\begin{array}{l}\text { Journal of the American } \\
\text { Soc. for Inf. Sc. (1986-1998) }\end{array}$ & 3 & $\begin{array}{l}\text { Biblioteconomía y } \\
\text { Documentación (LIS) }\end{array}$ \\
\hline Library Trends & 3 & Biblioteconomía y Doc. (LIS) \\
\hline First Monday & 2 & Computación \\
\hline Information \& Culture & 2 & Historia \\
\hline $\begin{array}{l}\text { Information Resources } \\
\text { Management Journal }\end{array}$ & 2 & $\begin{array}{l}\text { Biblioteconomía y } \\
\text { Documentación (LIS) }\end{array}$ \\
\hline $\begin{array}{l}\text { Int. Journal of Tech. and } \\
\text { Human Interaction }\end{array}$ & 2 & Computación \\
\hline $\begin{array}{l}\text { Int. Research: Journal of } \\
\text { Library and Inf. Science }\end{array}$ & 2 & $\begin{array}{l}\text { Biblioteconomía y } \\
\text { Documentación (LIS) }\end{array}$ \\
\hline Journal of Communication & 2 & Comunicación \\
\hline $\begin{array}{l}\text { Portal: Libraries and the } \\
\text { Academy }\end{array}$ & 2 & $\begin{array}{l}\text { Biblioteconomía y } \\
\text { Documentación (LIS) }\end{array}$ \\
\hline $\begin{array}{l}\text { Records Management } \\
\text { Journal }\end{array}$ & 2 & $\begin{array}{l}\text { Biblioteconomía y } \\
\text { Documentación (LIS) }\end{array}$ \\
\hline $\begin{array}{l}\text { Scientific and Technical } \\
\text { Information Processing }\end{array}$ & 2 & Computación \\
\hline
\end{tabular}

Tabla IV. Revistas más populares 
Su adscripción a cada grupo temático se comprobó en la información de las sedes web de cada una de las revistas. Asimismo, se consultó Scimago para verificar su adscripción. Del total de 89 títulos, 34 son propios de Biblioteconomía y Documentación. Este 38\% resalta frente a la escasa presencia de autores del área en el estudio de la filiación institucional.

\subsection{Resultados del análisis temático}

En cuanto a la distribución temática, se han contabilizado 48 categorías temáticas a las que se adscriben los artículos (Tabla V). Las áreas más productivas coinciden tanto con los resultados de la filiación de los autores como con los de las revistas: especialmente en la conexión entre la empresa y la aplicación de tecnologías de información. Los primeros son: gestión del conocimiento y capital intelectual y gestión en general, sistemas de información, aprendizaje organizacional y tecnologías de la información.

\begin{tabular}{|c|c|}
\hline Categorías temáticas & N. ${ }^{\circ}$ art. \\
\hline Gestión del Conocimiento y Capital Intelectual & 74 \\
\hline Sistemas de información & 26 \\
\hline Aprendizaje organizacional & 25 \\
\hline Gestión (Management) & 23 \\
\hline Tecnología de la Información & 19 \\
\hline Computación & 13 \\
\hline Bibliotecas & 12 \\
\hline Comunicación Corporativa & 10 \\
\hline Fundamentación Teórica LIS & 9 \\
\hline Bibliotecas académicas & 7 \\
\hline Comunicación & 7 \\
\hline Recuperación de Información ( IR) & 7 \\
\hline Cambio Organizacional & 6 \\
\hline Comportamiento organizacional & 6 \\
\hline Educación & 5 \\
\hline Inteligencia Artificial & 5 \\
\hline Información en la Web & 4 \\
\hline Organizaciones. Comportamiento Organizacional & 4 \\
\hline Cultura Organizacional & 3 \\
\hline Teoría del Caos & 3 \\
\hline Complejidad Computacional & 2 \\
\hline Complejidad Legal & 2 \\
\hline Información legal & 2 \\
\hline Administración Pública & 1 \\
\hline Archivística & 1 \\
\hline Bases de Datos & 1 \\
\hline Bibliotecas especializadas & 1 \\
\hline Búsqueda de Información & 1 \\
\hline Clasificación & 1 \\
\hline Complejidad Cognitiva & 1 \\
\hline Comportamiento informacional & 1 \\
\hline Clasificación & 1 \\
\hline Democracia & 1 \\
\hline Diseño de Páginas Web & 1 \\
\hline Filosofía & 1 \\
\hline Gestión de Bibliotecas & 1 \\
\hline
\end{tabular}

\begin{tabular}{ll}
\hline Gestión de la Información & 1 \\
\hline Gestión electrónica de documentos & 1 \\
\hline Hipertexto & 1 \\
\hline Liderazgo & 1 \\
\hline Procesamiento del Lenguaje Natural & 1 \\
\hline Propiedad Intelectual & 1 \\
\hline Redes de Información & 1 \\
\hline Servicio de referencia Digital & 1 \\
\hline Sistemas Open Source & 1 \\
\hline Sistemas Sociales & 1 \\
\hline Teoría de la Información & 1 \\
\hline Visualización & 1 \\
\hline
\end{tabular}

Tabla V. Categorías temáticas principales

Las bibliotecas como objeto de estudio o categoría temática ocupan el séptimo puesto con 12 artículos, mientras que la fundamentación teórica en Biblioteconomía y Documentación se da en 9 artículos. El resto de categorías afines desarrolladas son: bibliotecas universitarias 0 académicas - un área especialmente productiva en la aplicación de teorías organizacionales entre las que se encuentra la Complejidad-, las bibliotecas especializadas, la gestión de bibliotecas, la archivística o la gestión electrónica de documentos. En cuanto a los subtemas, una vez determinada la categoría principal del artículo se han señalado varias palabras clave para su adscripción temática. Se han contabilizado 239 subtemas. En la Tabla VI se han incluido solo aquellos que figuran más de una vez, añadiendo los 168 subtemas restantes a la categoría de otros.

\begin{tabular}{lr}
\hline Subtema & Frecuencia \\
\hline Teoría de la Complejidad & 47 \\
\hline Teoría del Caos & 40 \\
\hline Sistemas adaptativos complejos (CAS) & 16 \\
\hline Sistemas de Información & 16 \\
\hline Teoría de Sistemas & 14 \\
\hline Gestión del Conocimiento & 12 \\
\hline Complejidad & 8 \\
\hline Gestión del cambio & 8 \\
\hline Sistemas complejos & 8 \\
\hline Teoría de la Información & 7 \\
\hline Aprendizaje organizacional & 6 \\
\hline Gestión de bibliotecas & 6 \\
\hline Liderazgo & 6 \\
\hline Teoría de la Organización & 6 \\
\hline Toma de decisiones & 6 \\
\hline Transferencia de Conocimiento & 6 \\
\hline Aprendizaje & 5 \\
\hline Cambio organizacional & 5 \\
\hline Fundamentación teórica & 5 \\
\hline Recuperación de Información & 5 \\
\hline Teoría Organizacional & 5 \\
\hline Algoritmos & 4 \\
\hline Bibliotecas digitales & 4 \\
\hline Gestión de proyectos & 4 \\
\hline Innovación & \\
\hline & \\
\hline
\end{tabular}




\begin{tabular}{|c|c|}
\hline Internet & 4 \\
\hline Auto-Organización & 3 \\
\hline Bibliotecas & 3 \\
\hline Capital intelectual & 3 \\
\hline Ciencia de la Complejidad & 3 \\
\hline Creatividad & 3 \\
\hline Gestión de crisis & 3 \\
\hline Gestión estratégica & 3 \\
\hline Hipertexto & 3 \\
\hline Networking & 3 \\
\hline Postmodernismo & 3 \\
\hline Redes & 3 \\
\hline Redes sociales & 3 \\
\hline Activos intangibles & 2 \\
\hline Administraciones Públicas & 2 \\
\hline Autopoiesis & 2 \\
\hline Caos & 2 \\
\hline Comercio electrónico & 2 \\
\hline Comportamiento organizacional & 2 \\
\hline Computación & 2 \\
\hline Comunicación & 2 \\
\hline Conocimiento tácito & 2 \\
\hline Cynefin Framework & 2 \\
\hline Divulgación científica & 2 \\
\hline Epistemología & 2 \\
\hline Filosofía & 2 \\
\hline Física & 2 \\
\hline Gestión de la cadena de suministro & 2 \\
\hline Gestión de la Información & 2 \\
\hline Improvisación & 2 \\
\hline Información en la Web & 2 \\
\hline Ingeniería de Sistemas & 2 \\
\hline Inteligencia Artificial & 2 \\
\hline Inteligencia Competitiva & 2 \\
\hline Medición de la Complejidad & 2 \\
\hline Modelo basado en agentes & 2 \\
\hline Motores de búsqueda & 2 \\
\hline Organizaciones & 2 \\
\hline Relaciones públicas & 2 \\
\hline Sector público & 2 \\
\hline Sistemas & 2 \\
\hline Software & 2 \\
\hline Tecnología & 2 \\
\hline Tecnologías de la Comunicación & 2 \\
\hline Teoría de la Decisión & 2 \\
\hline Web 2.0 & 2 \\
\hline Otros & 68 \\
\hline
\end{tabular}

Tabla VI. Distribución de los subtemas

En cuanto a la presencia del área de Biblioteconomía y Documentación en las palabras clave, solo figuran de manera explícita gestión de bibliotecas (6), bibliotecas (4) y bibliotecas digitales (3). Entre las 168 palabras clave restantes se encuentran además subdisciplinas como alfabetización informacional, búsqueda de información, clasificación, diseño de bibliotecas y bibliometría.

Al igual que en análisis previos un escaso número de palabras clave concentra el $32 \%$ de los artículos producidos. Entre las palabras clave que más se repiten, destacan las específicas de las teorías asociadas con la Complejidad (47), Caos (40) y la Teoría de Sistemas (14) y de Sistemas Complejos (16). Nuevamente se aprecia la influencia de la temática sobre Sistemas de Información en empresas (16) y la Gestión en todas sus perspectivas, desde la Gestión del Conocimiento, pasando por el comportamiento organizacional, Liderazgo o la Toma de decisiones en la Organización.

\section{Discusión}

Las dificultades para delimitar el corpus teórico de la Complejidad son las mismas que para estudiar su uso en la producción bibliográfica. La lectura de los artículos y su análisis exhaustivo ha permitido extraer elementos de juicio relevantes sobre su presencia en Biblioteconomía y Documentación.

En cuanto a la presencia de autores del área, es significativo que, del total de 566 autores, solo en 20 artículos sus autores se vinculan a departamentos asociados al área de Biblioteconomía y Documentación. Entre ellos, destaca la obra de David Bawden, Organised complexity, meaning and understanding (2007), en la que incluye los sistemas autorganizativos para delimitar su teoría unificada de información. Otra obra de interés se adscribe al campo del comportamiento informacional (Información Behaviour), se trata de Towards a conceptual framework for provider information behaviour (2013) de Richmond Davies y Dorothy Williams, en la que se trata de proveer un marco teórico multidisciplinar, incluyendo la Complejidad. Se cita de manera expresa la obra de Mitleton-Kelly (2003) sobre sistemas complejos y Organización titulada, Complex Systems and Evolutionary Perspectives on Organisations: The Application of Complexity Theory to Organisations. Otro uso aislado es la aplicación de la Teoría del Caos al ámbito de la Bibliometría por P. C. R Borges (2002), ligado a la Universidad de Brasilia.

Las demás obras vinculadas en su filiación institucional a bibliotecas o departamentos LIS tienen un denominador común: su aplicación al ámbito de la Gestión de bibliotecas y al análisis del comportamiento organizacional. Resulta de interés la obra Chaos theory, economics and information: the implications for strategic decision making de T. Hayward y J. Preston (1999) en la que aplican la Teoría del Caos para la toma de decisiones, incluyendo de manera expresa la obra de Stacey (1993), Strategy as order emerging from chaos o la de H. Simon (1962), Admi- 
nistrative Behaviour: A Study of Decision Making Processes in Administrative Organisations.

El análisis de todas las filiaciones institucionales detectó la confluencia de departamentos heterogéneos entre Sistemas de Información y el ámbito de la Decisión y la empresa. En el caso de autores españoles, no figura ningún autor español vinculado a ningún departamento de Biblioteconomía y Documentación. Tan solo se encuentran dos departamentos de Organización de Empresas de la Universidad Rey Juan Carlos y la Universidad Autónoma de Madrid. Ambas aportaciones se realizaron en el Journal of Intellectual Capital, fuente que, si bien cubre la temática de Gestión del Conocimiento, es de marcada tendencia de Empresa y Finanzas.

El análisis de las revistas demuestra el mismo patrón: la abundancia de fuentes asociadas al ámbito de la Gestión y los Sistemas de Información. Muchas se adscriben a varias categorías temáticas en los rankings de publicaciones como Scimago. Destacan en nuestro ámbito, las revistas asociadas a la Gestión del Conocimiento. También es destacable la presencia de revistas que aceptan propuestas más teóricas como Journal of Documentation o Journal of Classification. Los resultados demuestran el predominio de fuentes y trabajos anglosajones. De los 303 artículos analizados, 298 son en lengua inglesa, estando representados el portugués (3), alemán (1) y francés de manera simbólica.

En cuanto a los resultados del análisis temático, el uso de la Teoría de Complejidad y la Teoría del Caos en el marco de la Gestión del Conocimiento es el mayoritario. La mayoría de las aportaciones se enfocan a proveer un cuerpo teórico para posteriormente analizar los cambios organizacionales o diseñar sistemas. Generalmente, en el marco de la organización compleja se estudian los procesos de transferencia de conocimiento, poniendo énfasis en su medición y evaluación.

Es revelador el uso que se ha podido detectar de la presencia de la teoría de manera operacional, como marco metodológico u operativo de estudio de problemas y organizaciones, y de cualquier sistema emergente. Esta característica es común en el escaso número de artículos adscritos al área de Biblioteconomía y Documentación. Por ello, llaman la atención aquellos etiquetados bajo el epígrafe de "Fundamentación Teórica LIS", que tratan de enriquecer el cuerpo teórico con el uso de estos aportes científicos. Así, al margen de la obra citada de Bawden (2007), y la co-escrita con Robinson titulada Waiting for Carnot: Information and complexity (Bawden y Robinson, 2015), destaca el trabajo de Bonna Jo- nes (2008) titulado Reductionism and library and information science philosophy en el que se menciona a la Teoría de la Complejidad como medio para luchar contra el reduccionismo teórico en la Biblioteconomía y Documentación.

Por último, el análisis de temas y subtemas ha puesto de manifiesto los diferentes enfoques mediante los que se pueden abordar las llamadas "Ciencias de la Complejidad". Desde la Teoría de Sistemas complejos, a la introducción de la noción de caos y sistemas no-lineales pasando por los conceptos de auto-organización y emergencia. Todas estas nociones dificultan un análisis homogéneo de estas teorías.

\section{Conclusiones}

Los resultados del análisis muestran un uso muy moderado de la Teoría del Caos o de la Complejidad en el ámbito de la Biblioteconomía y Documentación. Sus usos más comunes son los aplicados a estudios de Gestión y Comportamiento organizacional de los centros. Se ha observado una influencia del ámbito de la Economía y Empresa en la producción científica analizada seguida de la visión desde la Computación y el diseño de Sistemas.

Si bien en algunos estudios se intentan construir macroteorías y Teorías Unificadas basadas o inspiradas en la Complejidad, el uso detectado es marcadamente operacional. Esta conclusión verifica las opiniones de Heylighen, Cilliers, Gershenson sobre su uso especializado y restrictivo y una falta de mayor asimilación por parte de la Comunidad para su uso a nivel de fundamentación teórica.

A pesar de este intento en enmarcar la producción científica en LIS, estudios futuros como el análisis de la bibliografía pueden aportar cuál es el alcance e impacto real de la Complejidad y si estas obras se utilizaron como elemento sustancial o como parte de la revisión bibliográfica.

\section{Agradecimientos}

El presente artículo es continuación del marco teórico empleado en la tesis doctoral titulada "Estudio de la complejidad estructural y dinámica de la Biblioteca Digital Universitaria: un modelo de cambio basado en el Conocimiento" (2014), dirigida por Dra. Mercedes Caridad Sebastián y Dr. Antonio Bereijo Martínez (q.e.p.d) y financiada por el Ministerio de Educación a través del Programa de Formación de Profesorado Universitario (FPU).

\section{Referencias}

Bawden, David (2007). Organized complexity, meaning and understanding: An approach to a unified view of information for information science. // Aslib Proceedings. 59: $4 / 5,307-327$ 
Bawden, David; Robinson, Lyn (2015). Waiting for Carnot: Information and complexity. // Journal of the Association for Information Science and Technology. 66:11.

Bereijo Martínez, A (2012). La sobriedad de factores en las Ciencias de la Documentación. // González, W.J. (Ed.). Las Ciencias de la Complejidad: vertiente dinámica de las Ciencias de Diseño y sobriedad de factores. A Coruña: Netbiblio. 267-284.

Borges, Paulo Cesar Rodrigues (2002). Métodos quantitativos de apoio à bibliometria: a pesquisa operacional pode ser uma alternativa. // Ciência da informação 31:3, 5-17.

Brookes, Bertram C (1980). The foundations of information science Part I. Philosophical aspects. // Journal of information science. 2:3-4, 125-133.

Davies, Richmond; Williams, Dorothy (2013). Towards a conceptual framework for provider information behavior. // Journal of Documentation. 69: 4, 545-566.

Delic, Kemal; A.; Dum, Ralph (2006). On the emerging Future of Complexity Sciences. // ACM Ubiquity. 2.

Gilpin, Dawn R; Murphy, Priscila J (2008). Crisis management in a complex world. Oxford: Oxford University Press.

Gilstrap, Donald L (2009). A complex systems framework for research on leadership and organizational dynamics in academic libraries. // portal: Libraries and the Academy. 9:1, 57-77.

Gleick, James (1987). Chaos: Making a New Science. Elmsford: Pergamon Press.

González, Wenceslao J (2012). Las Ciencias de Diseño en cuanto a Ciencias de Complejidad: Análisis de la Economía, Documentación y Comunicación. // González, W.J (Ed.). Las Ciencias de la Complejidad: vertiente dinánica de las Ciencias de Diseño y sobriedad de factores. A Coruña: Netbiblio. 7-20.

González, Wenceslao. J (2013). Los límites del universalismo metodológico: el problema de la Complejidad. // Naturaleza y libertad: Revista de estudios interdisciplinares. 2, 61-89.

Grover, Robert, et al (1997). The wind beneath our wings: chaos theory and the butterfly effect in curriculum design. // Journal of education for library and information science. 38:4 268-282.

Hayward, T.; Preston, J. (1999). Chaos theory, economics and information: the implications for strategic decision making. // Journal of Information Science. 25: 3, 173-182.

Heylighen, Francis; Cilliers,Paul; Gershenson,Carlos (2007). Complexity and philosophy. // Bogg, J.; R. Geyer (eds.) Complexity, Science and Society. Oxford: Radcliffe Publishing.

Hjørland, Birger (1998). Theory and metatheory of information science: a new interpretation. // Journal of documentation.54:5, 606-621.

Jones, Bonna (2008). Reductionism and library and information science philosophy. // Journal of Documentation $64: 4,482-495$.

Layzell Ward, P. (2000). An overview of the literature of management and of information and library services management 1999. // Library Management. 21:3, 128-152.

Layzell Ward, P. (2002). Management and the management of information, knowledge-based and library services 2001. // Library Management. 23:3, 135-165.

Line, M. B. (2001). Process think: Winning perspectives for business change in the information age. // Journal of Documentation. 57:2, 306-307.

Line,M. B (1995). Is strategic planning outmoded?. // Alexandria $1: 3,135-137$.

Luhmann, Nikles (1998). Sistemas sociales: lineamientos para una teoría general. Barcelona: Anthropos, 1998.
Lyytinen, K; Newman, M (2008). Explaining information systems change: a punctuated socio-technical change model. // European Journal of Information Systems. 17:6, 589-613.

Martínez Cardama, S (2014). Estudio de la complejidad estructural y dinámica de la Biblioteca Digital Universitaria: un modelo de cambio basado en el Conocimiento. Madrid: Universidad Carlos III de Madrid.

Maturana, Humberto R; Varela, Francisco J. (1987). The Tree of Knowledge: The Biological Roots of Human. Boston: New Science Library/Shambhala Publications.

Mitleton-Kelly,Eve (2003). Complex systems and evolutionary perspectives on organisations: the application of complexity theory to organisations. Oxford: Elsevier Science.

Nolin, Jan; Åström, Fredik (2010). Turning weakness into strength: Strategies for future LIS. // Journal of Documentation. 66:1, 7-27.

Pettigrew, Karen E.; McKechnie, Lynne EF (2001). The use of theory in information science research. // Journal of the American Society for Information Science and Technology. 52:1, 62-73.

Pienaar, H. et al.(1999b) Launching transformations at the Academic Information Service, University of Pretoria.// IATUL Proceedings (New Series). 8.

Pienaar, H.; et al. (1999a), Organisational transformation at an academic information service. // Library Management. 20: 5, 266-272.

Rodríguez Zoya, Leonardo G; Leónidas Aguirre, Julio (2011). Teorías de la complejidad y ciencias sociales: nuevas estrategias epistemológicas y metodológicas. // Nómadas: Revista crítica de ciencias sociales y jurídicas. $30: 2$

Saarti, J.; Juntunen, A. (2011a). The benefits of a quality management system. // Library Management. 32:3, 183:190.

Saarti, J.; Juntunen, A. (2011b). Bringing out the best of everyone. // Library Management. 32:8, 579-588

Saracevic, Tefko (1995). Interdisciplinary nature of information science. // Ciência da informação. 24:1, 36-41

Shannon, C.E.; Weaver, W (1949). The mathematical theory of communication. Urbana, IL: University of Illinois Press

Simon, Herbert A (1962). The architecture of complexity. // Proceedings of the American philosophical society. 106:6, 467-482.

Stacey, R. (1993). Strategy as Order Emerging from Chaos. // Long range planning. 26:1.10-17.

Tredinnick, Luke (2009). Complexity theory and the web // Journal of documentation. 65:5, 797-816.

Van Maanen, John (1988). Different strokes: Qualitative research in the Administrative Science Quarterly from 1956 to 1996. // Van Maanen, J. (Ed.). Qualitative studies of organizations. Thousand Oaks, Ca: SAGE. 9-32

Von Bertalanffy, Ludwig (1968). General system theory: Foundations, development, applications. New York: Brazilier.

Wiener, N. (1948). Cybernetics, or control and communication in the animal and the machine. New York: John Wiley \& Sons.

Woermann, M. (2016). Bridging complexity and post-structuralism: Insights and implications. Switzerland: Springer.

Enviado: 2017-05-09. Segunda versión: 2017-09-10 Aceptado: 2017-09-12. 